\title{
Sexually transmitted disease control
}

\author{
PAUL R GULLY, MB, FFCM, FRCPC
}

$\mathrm{T}$ HE LABORATORY CENTRE FOR DISEASE CONTROL (LCDC) is the national centre for preserving and improving public health through the identification, investigation, control and prevention of disease in humans. The most common reportable diseases are sexually transmitted (STD). Data on the national picture of STD in Canada have been collected since the early 1920s. Interest and concern with the control of STD has varied, but revived in the 1980s most probably due to the emergence of human immunodeficiency virus (HIV) infection and the acquired immune deficiency syndrome (AIDS) which is primarily an STD.

The Division of Sexually Transmitted Disease Control of the LCDC was formed in late 1987 with Dr Gordon Jessamine as chief. It is the division's role to describe the current status of STD (excluding HIV infection and AIDS) in Canada by routine and nonroutine surveillance, to initiate research into surveillance and control and to support control efforts nationwide. Canadian guidelines for the diagnosis, management and treatment of STD are produced in collaboration with the Provincial/Territorial Directors of STD Control, STD experts, organizations and the National Laboratory for STD of the LCDC.

\section{EPIDEMIOLOGY}

The current epidemiology of STD is derived from analyses of reports of notifiable communicable diseases from the provinces and territories. There is also a process to implement gradually the collection of caseby-case information on reports of STD. The publication of case definitions for surveillance of communicable diseases including STD has assisted the reporting process (1).

The reported rates of gonorrhoea have dropped significantly since the beginning of the 1980 s. The reason for this precipitous fall may be due to a number of

Correspondence and reprints: The Laboratory Centre for Disease Control, Ottawa, Ontario K1A OL2 factors such as improved control through treatment and contact tracing as well as primary and secondary prevention efforts stimulated by the advent of HIV infection and AIDS. Although the highest reporting rates are in the age group 20 to 24 years, the group with the highest rates of gonorrhoea are 15- to 19-year-old women.

Knowledge of the epidemiology of chlamydial infection in Canada is poor as reporting of this disease is relatively new in a number of jurisdictions. There has been relatively recent recognition that Chlamydia trachomatis is not only a very important cause of nongonococcal urethritis and mucopurulent cervicitis but that it can also cause pelvic inflammatory disease, ectopic pregnancy and infertility.

Chlamydial infections became nationally notifiable in 1990; we are now able to collect, for the first time, reports of chlamydial infection from most provinces and territories. In addition, there are historical data from some jurisdictions: in Manitoba, Quebec and Nova Scotia it is evident that the rates of reported chlamydia are falling. Overall Canadian rates are two to five times higher than those for gonorrhoea.

Reported rates of chlamydia are highest in women aged 15 to 19 years (1579 per 100,000 population) where long term sequelae are particularly important. It should always be remembered that the denominator for calculating rates should ideally take account of the fact that only a proportion of adolescents are sexually active. The reported rates of infection in young women are definitely an underestimate.

It is difficult to make comparisons between areas of the country due to different reporting and screening practices. As a majority of cases of chlamydial infection in man and women is asymptomatic, the amount of screening activity and the groups being screened will influence reporting rates. The Northwest Territories have reported rates far higher than the norm across Canada. This may be a reflection of higher rates of disease but also of appropriate identification strategies. 
The explanation for high rates of chlamydial infection contrasted to the marked drop in gonococcal infections could be explained if treatment and contact tracing of gonorrhoea have been carried out more aggressively than for chlamydia; preventive activities would presumably affect both diseases. It is likely that control activities have concentrated on gonorrhoea, most recently on antimicrobial resistant gonorrhoea and syphilis. The burden of chlamydia is affecting a much wider population than that affected by gonorrhoea which is likely to be maintained in 'core groups'. The division is collaborating with the Chlamydia Section of National Laboratory of STD in research designed to better describe the prevalence (sic) of infection in high risk groups.

The Expert Interdisciplinary Advisory Committee on Sexually Transmitted Diseases in Children and Youths (EIAC-STD) ended its five year mandate in March 1991. The committee sponsored research into STD and was supported by the Family Violence Initiative of Health and Welfare Canada. The national study to determine clinical and biological factors influencing Neisseria gonorrhoeae infections and treatment in children and adults of the National Laboratory for STD in 1988-89 was one example of this research. The Division of STD Control is collaborating with the National Laboratory for STD in a continuation of the national study and it is hoped, in the next two years, to obtain epidemiological as well as biological information on a sample of nonresistant gonorrhoea isolates as well as all resistant isolates and all isolates from children from across Canada. The Division of STD Control is also collaborating with the National Laboratory for STD Control and the Ontario Ministry of Health in a study of penicillinase-producing gonorrhoea cases in Metro Toronto.

It is extremely important to collect data on the treatment of STD by physicians especially given the present problem of antimicrobial resistant gonorrhoea. A significant proportion (greater than 3\%) of cases of gonorrhoea In British Columbia, Alberta, Manitoba, Ontario and Quebec, are now resistant to at least penicillin. The Division is collaborating with the College of Family Physicians of Canada to document the treatment of STD by primary care physicians.

The first set of guidelines for the management, treatment and diagnosis of STD in children and adults were also sponsored by the EIAC-STD Committee, these guidelines have been widely distributed and are in the process of revision. Interim guidelines for the treatment of uncomplicated gonorrhoea were published recently (4).

The ELAC-STD also collaborated with other agencies in the Canada Youth and AIDs Study. Secondary analysis of data from this study is still being carried out, in part by the Division and is providing an important insight into the behaviour of young people. It will be vital to repeat such a survey to ascertain whether behaviour leading to STD has changed since 1988.

The reported incidence of infectious syphilis in this country is low. It remains to be seen whether there will be a resurgence of congenital and infectious syphilis as has been reported from the United States associated with the exchange of sex for drugs. It has been recognized that hepatitis B is in part an STD. This issue was discussed in a supplement to The Canadian Journal of Infectious Diseases (3).

There are also, of course, other STD which are not reportable and for which it is very difficult to ascertain data on incidence or prevalence. Undoubtedly, genital warts (caused by the human papillomavirus) are a common presentation in individuals visiting STD clinics ( 4 to $6 \%$ of STD visits by males and females 15 to 24 years old to selected Canadian clinics) (Division of STD Control, LCDC, unpublished data). The relationship between human papillomavirus infection, warts and later genital cancer, especially cancer of the cervix is, however, complex. The importance of regular Pap smears in individuals with a history of genital warts should be emphasized. Herpes simplex virus infections are also common but the incidence of primary genital infection is difficult to assess. Trichomonas vaginalis infection is also sexually transmitted. It may be possible to ascertain from provincial and territorial medical plan data the number of visits for these infections to physicians and therefore to gauge the size of these problems. Hospital admission data are not very helpful for STD but have been used to assess the size of the problem of pelvic inflammatory disease and ectoplc pregnancy and will also be used In the future to repeat previous work assessing the cost of sequelae of chlamydial infection.

\section{CHALLENGES}

In short our challenges are: the refining of surveillance methods, estimation of the overall impact of STD including HIV infection and AIDS; and the evaluation of control methods especially contact tracing.

\section{REFERENCES}

1. Canadian Communicable Disease Surveillance System: Disease-specific case definitions and surveillance methods. Can Dis Weekly Rep 1991;17(Supplement 3).

2. Gully PR, Rwetsiba D. Trends in gonorrhoea in Canada: 1980-1989. Can Dis Weekly Rep 1991;17:105-9.

3. Hepatitis B: Canada's overlooked STD. Implications for health care policy. Can J Infect Dis 1991;2 (Suppl A): 1A-37A.

4. Interim guidelines for the treatment of uncomplicated gonococcal infection. Can Dis Weekly Rep 1991;17:281-2. 


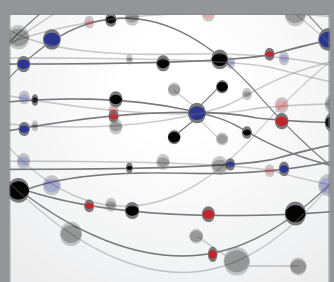

The Scientific World Journal
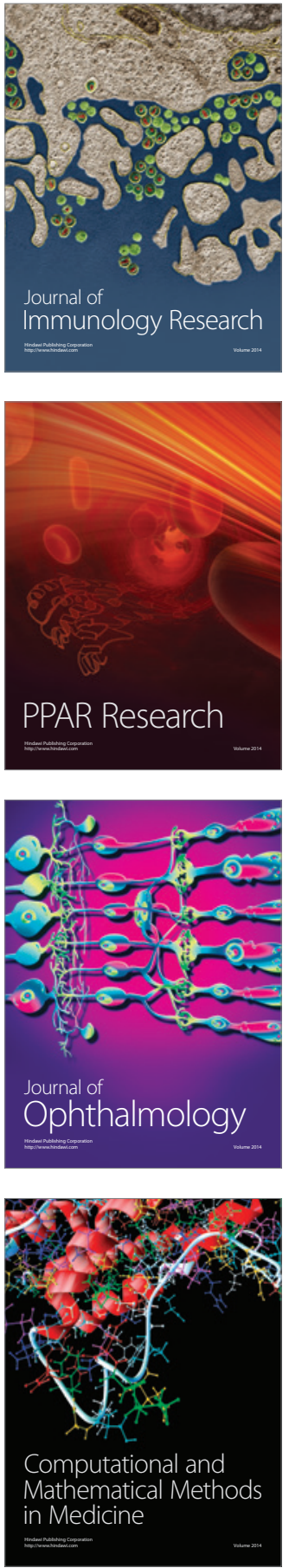

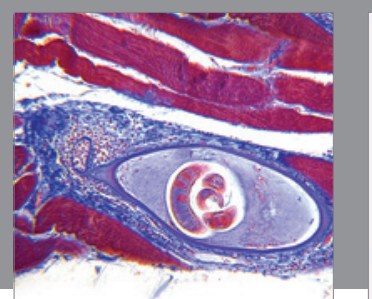

Gastroenterology Research and Practice

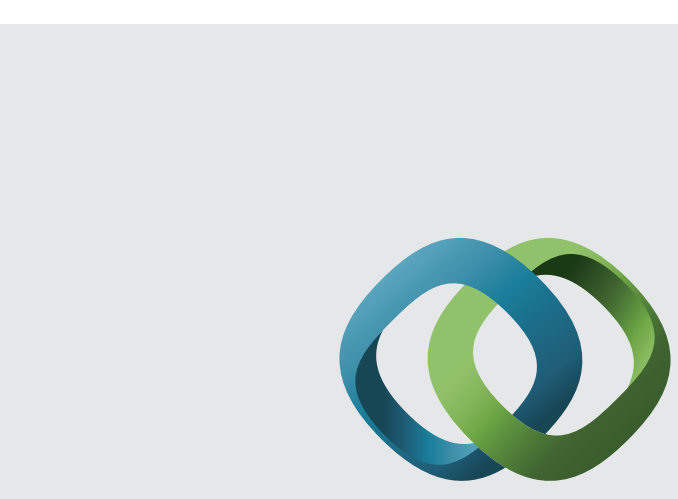

\section{Hindawi}

Submit your manuscripts at

http://www.hindawi.com
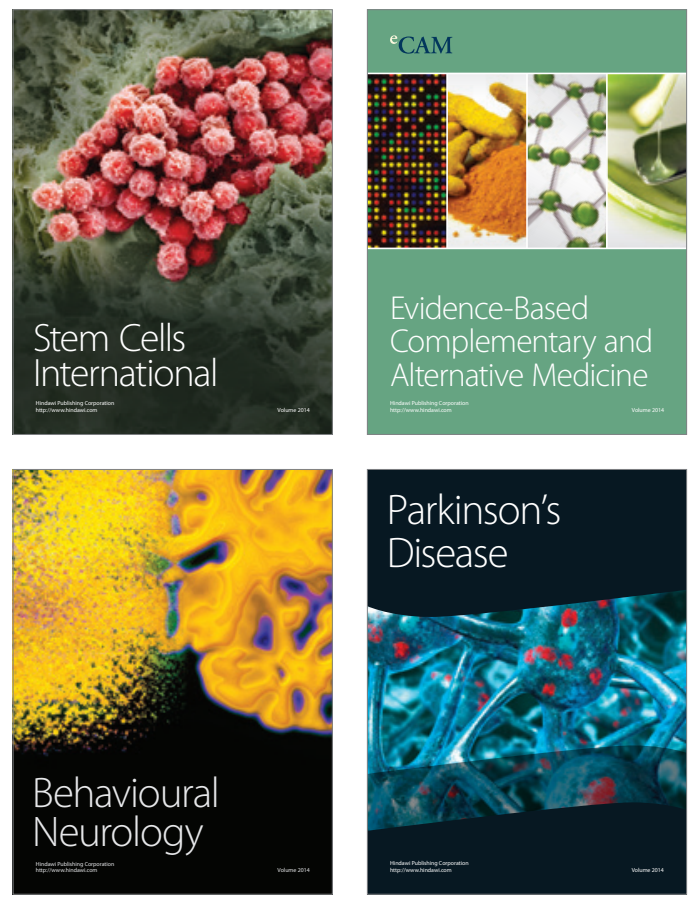
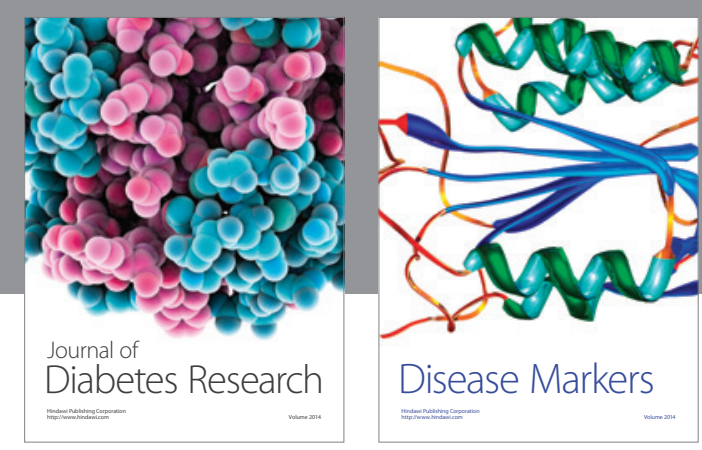

Disease Markers
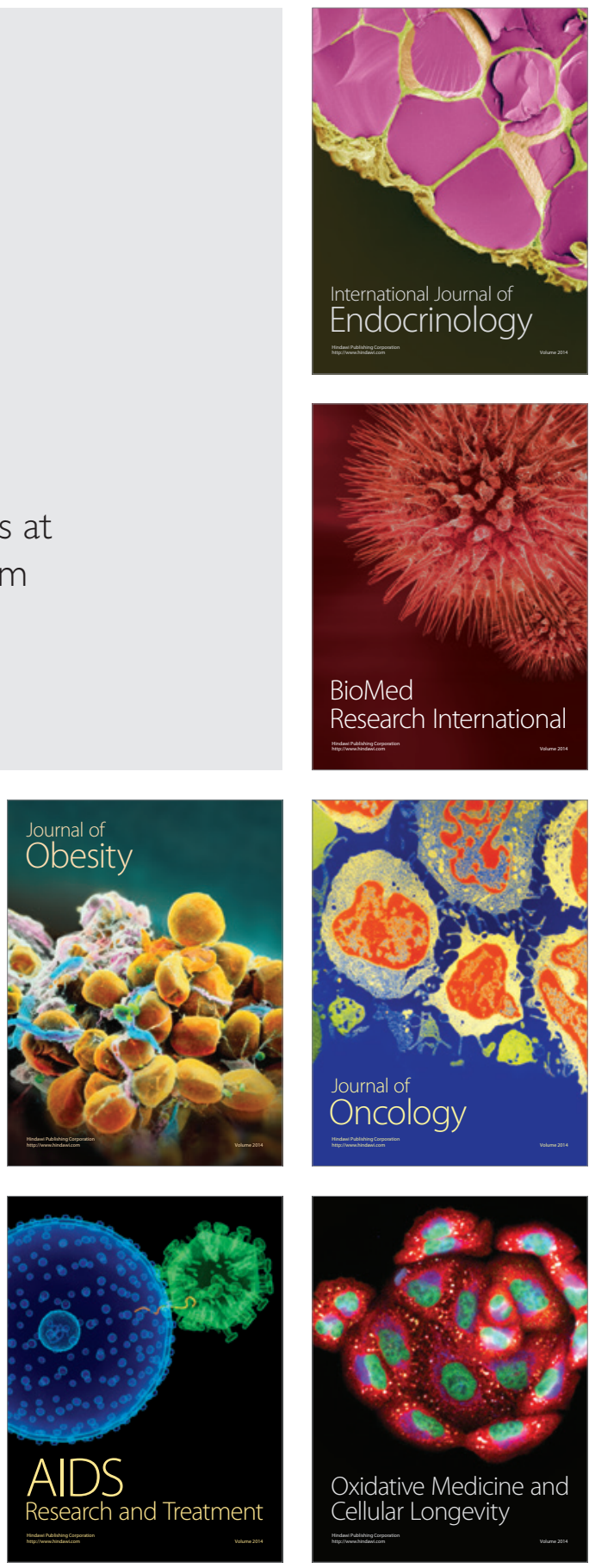